\title{
Beta Amyloid, Brain-Derived Growth Factor and Cognitive Function in Circadian Rhythm Disturbances
}

\author{
Chang-Quan Huang*, Run-Feng Zhang, Qing-Xiu Liu, Hu Xiang, Ji Sheng Wang and Xiao-Dong Peng \\ Department of Geriatrics, Chongqing Medical University, China
}

*Corresponding author: Chang-Quan Huang, Department of Geriatrics, The third hospital of mianyang, Chongqing Medical University, China.

Received Date: February 18, 2020

Published Date: March 05, 2020

\begin{abstract}
s rhythm disturbances (CRDs). $\mathrm{BDNF}$ and $\mathrm{A} \beta$ levels were determined ELISA Kit.

Result: In CRDs, there were cognitive function declines, BDNF decreased and A $\beta$ increased.

Conclusion: CRDs can lead to cognitive function declines; it was related to BDNF decreased and A $\beta$ increased.
\end{abstract}

Objective: this study observes the changes of cognitive function, $\beta$-amyloid peptide (A $\beta$ ) and Brain-derived growth factor (BDNF) in circadian

Method: Animal models of CRDs induced by light-dark cycles in different periods. Cognitive function in mice was detected by water maze test.

Keywords: circadian rhythm disturbances; cognitive function; Brain-derived growth factor; $\beta$-amyloid peptide

\section{Introduction}

Several studies have demonstrated that circadian rhythm disturbances (CRDs) can lead to cognitive function declines [1]. The morbidity and prevalence of dementia (including Alzheimer's disease) in people with CRDs are higher than those in the general population [2-4]. Even CRDs can lead to shorter life expectancy [24]. The biological effects of CRDs are mostly concentrated in clinical studies, mainly in the observation of phenomena, and research on relevant mechanisms has been few done [1,7]. Improving CRDs is the best way to significantly reduce its harm, however, improving CRDs is very difficult and the method is very limited. Therefore, it is very important to elucidate the mechanism of the effects of CRDs on organisms.

It has been documented that Brain-derived growth factor (BDNF) expression is reduced in the brains of Alzheimer's disease (AD) [8-13]. Patients AD are characterized by the accumulation of $\beta$-amyloid peptide $(A \beta)$ within the hippocampus along with hyperphosphorylated [8-13]. A $\beta$ level is decreased by BDNF in primary neuronal cultures, whereas it is elevated by BDNF deprivation $[14,15]$. BDNF may have a protective role against AD pathogenesis. The beneficial effect of BDNF administration has been shown to increase cognitive function in impaired animals, and studies in AD models show that BDNF has a neuroprotective effect against $A \beta$ toxicity [16-18]. Neuronal cell growth is modulated by factors such as BDNF [19-21]. BDNF is highly concentrated in the hippocampus, important in synaptic plasticity, and contributes to neurogenesis in the dentate gyrus16-18. BDNF plays a pivotal role in age-related memory impairments and is associated with agerelated atrophy of the hippocampus [16-18]. Previous studies have reported that serum $\mathrm{BDNF}$ levels are reduced in $\mathrm{AD}[17,19]$. A study of neuronal cell cultures found that amyloid peptide at sublethal concentrations interfered with neuronal plasticity mediated by BDNF signaling cascade [22].

The mechanisms underlying cognitive function declines or AD disease caused by CRDs has been unclear2. In individuals 
with CRDs disorders, BDNF decreases or A $\beta$ increases, it's only a hypothesis $[2,7]$. If this hypothesis can be confirmed, this may be a mechanism for cognitive impairment and increased Alzheimer's disease caused by CRDs. In this study, we tested the BDNF and A $\beta$ content in hippocampus of experimental animal models of CRDs to verify this hypothesis.

\section{Experiment}

\section{Experimental animal and establishment of circadian rhythm model}

90 C57BL/6 Male mice (Animal experiment center of Sichuan University, Chengdu, China), weighing 20-22 g (8 weeks old), were used in the experiments. The mice were maintained under standard laboratory conditions, and were housed in acrylic cages $(20 \mathrm{~cm} 27$ $\mathrm{cm} 12 \mathrm{~cm}$ ) under an artificial 12-hour light/ dark cycle at a controlled temperature $\left(22 \pm 2^{\circ} \mathrm{C}\right)$ and humidity $(60 \pm 10 \%)$, with free access to water and food unless specified otherwise departmental room for 7 days to ensure adaptation to the new environment. All animal procedures were conducted under the regulations of the Sichuan University Animal Care and Use Committee, Chengdu, China. All applicable international, national, and/or institutional guidelines for the care and use of animals were followed.

Light-dark cycle was as a method of studying the circadian rhythm [23]. Firstly, the mice were randomly divided into three groups: Light-dark cycle 12/12 hour group, Light-dark cycle 3/3 hour group and Light-dark cycle 22/22 hour group, each group included 30 mice. Light-dark cycle 12/12 hour group was as sham control, the other groups were as CRDs group. After grouping, mice in each group were fed for 12 weeks.

\section{Cognitive function analysis}

Water maze experiment was used to test cognitive function [24]. The water-maze tank was placed with four external visual cues and filled with water containing a nontoxic white color. The temperature of the water was maintained by $23-25^{\circ} \mathrm{C}$. A white platform $(10 \mathrm{~cm}$ in diameter and $30 \mathrm{~cm}$ in height) was placed in one of the quadrants with equal area and submerged $2 \mathrm{~cm}$ below the water surface. During each trial session, the escape latency time spent to find the hidden platform was monitored by a video tracking system. During the four subsequent days of training the mice were given three trials per day with the submerged platform in the pool. When the mouse located on the plated platform, it was allowed to remain on it for an additional $10 \mathrm{sec}$. If the mouse did not find the hidden platform within $120 \mathrm{sec}$, the mouse was guided to the platform and permitted to remain on it for an additional $10 \mathrm{sec}$. On the last day, the hidden platform was removed from the watermaze tank and probe test was performed. Mice were allowed to swim for $90 \mathrm{sec}$ and the staying time in the maze quadrant where the platform had previously been located was recorded.

\section{BDNF and $A \beta$ analysis}

BDNF and $A \beta$ levels were determined as previously described using the mice BDNF and A $\beta$ ELISA Kit (Thermo Fisher Scientific) [25]. Briefly, from three subregions of the hippocampus formation (CA1, CA3, and dentate gyrus), sample tissue was preservative and treated according to ELISA Kit instructions. As the tissue punches contained less tissue than the entire hippocampus, it was homogenized in $250 \mu$ l of Lysis buffer (Promega) to prevent dilution and the full $100 \mu \mathrm{l}$ of sample specified in the kit was added to each well. As tissue weight could not be obtained, total protein levels were measured by Bradford assay. BDNF and $A \beta$ concentration is expressed as ng/g protein.

\section{Data analysis}

Values from detected Morris Water Maze Tests, BDNF and A $\beta$ level are reported as means \pm SE. Statistical analyses include ANOVA (Comparison among the three groups) and Bonferroni t-tests (The comparison between the two groups). The overall level of statistical significance was $\mathrm{p}<0.05$.

\section{Results}

\section{Comparison of cognitive function (Spatial learning and memory in the Morris water maze test)}

Comparison of cognitive function see Table 1. Among sham group, Light-dark cycle 22/22 hour group and Light-dark cycle $3 / 3$ hour group, the time to reach the platform was significantly difference. The time to reach the platform in sham group was significantly shorter than that the other group. The time to reach the platform in sham group was significantly shorter than the other two groups, but that in the other groups was no significantly difference. The comparison between each group, the time taken to find the hidden platform was the same as that of it the time to reach the platform (Table 1).

Table1: Comparison of cognitive function (Spatial learning and memory in the Morris water maze test) among three groups: 12/12 hour group, 3/3 hour group and 22/22 hour group (Mean $\pm S D, n=30$ ).

\begin{tabular}{|c|c|c|}
\hline Group & The Time to Reach the Platform & The Time Taken to Find the Hidden Platform \\
\hline $3 / 3$ hour group & $11.68 \pm 2.22^{* *}$ & $25.32 \pm 4.91^{* *}$ \\
\hline $12 / 12$ hour group & $12.27 \pm 3.04^{* *}$ & $28.67 \pm 4.23^{* *}$ \\
\hline $22 / 22$ hour group & $8.57 \pm 1.93$ & $17.32 \pm 3.76$ \\
\hline
\end{tabular}

Compared 6/6 hour group ${ }^{*} P<0.05,{ }^{* *} P<0.01$. Compared 12/12 hour group ${ }^{\#} P<0.05,{ }^{\#} P<0.01$. 


\section{Comparison of BDNF in hippocampal}

Comparison of BDNF in hippocampal see Figure 1. Among sham group, Light-dark cycle $3 / 3$ hour group and Light-dark cycle 22/22 hour group, the BDNF levels in hippocampal were significantly difference. The BDNF level in hippocampal in sham group was higher significantly shorter than that the other group. The BDNF level in hippocampal in sham group was significantly higher than the other two groups, but that in the other groups was no significantly difference (Figure 1).

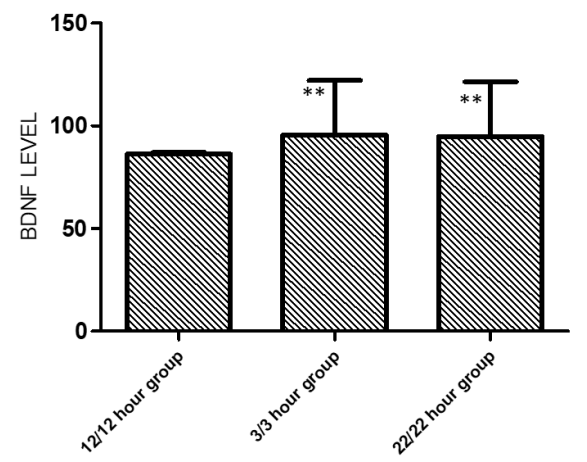

Figure 1 Comparison of Brain-derived growth factor (BDNF) in hippocampal among the three groups: 12/12-hour group, 3/3 hour group and 22/22 hour group.

Compared 6/6-hour group ${ }^{*} P<0.05,{ }^{* *} P<0.01$. Compared 12/12 hour group \#P<0.05, \#\#<0.01.

\section{Comparison of $A \beta$ in hippocampal}

Comparison of $A \beta$ in hippocampal see Figure 2. Among sham group, Light-dark cycle $3 / 3$ hour group and Light-dark cycle $22 / 22$ hour group, the $A \beta$ in levels hippocampal were significantly difference. The $A \beta$ level in hippocampal in sham group was lower significantly shorter than that the other group. The $A \beta$ level in hippocampal in sham group was significantly higher than the other two groups, but that in the other groups was no significantly difference (Figure 2).

Figure 2 Comparison of $\beta$-amyloid peptide $(A \beta)$ in hippocampal among the three groups: 12/12 hour group, 3/3 hour group and 22/22 hour group.

Compared 6/6 hour group ${ }^{*} \mathrm{P}<0.05,{ }^{* *} \mathrm{P}<0.01$. Compared 12/12 hour group \#P<0.05, \#\# $<0.01$.

\section{Discussion}

In the present study, we demonstrated that in CRDs mice cognitive function declines, BDNF levels in hippocampal decreased and the $A \beta$ in levels hippocampal increased. Biological rhythms include annual, monthly and circadian rhythm rhythms [26]. Women's menstrual cycle is a manifestation of monthly rhythm. Circadian rhythm rhythms are the most significant biological rhythm affecting organisms [27]. Therefore, it is also the biological rhythm that is the most concerned at present [27]. In this study, different light-dark cycles were used to establish the circadian rhythm model, it's also widely accepted [28,29]. First, behavioral changes and different levels of hippocampal protein in animal models with different light-dark cycles confirm that this method can be used as a method to establish animal models with different 
circadian rhythms. The cycle of light and darkness is usually 24 hours, and the time of light and darkness may be uneven (unequal 12-hour light and 12-hour darkness) in different areas, not exactly 24 hours, but the mutation did not exceed one hour. Light-dark cycle $3 / 3$ hour group and Light-dark cycle 22/22 hour, and their cycles are 6 hours and 44 hours, respectively. Compared with the other two groups, the light-dark cycle 12/12 can reflect the effects of CRDs on organisms. Second, compared with the other two groups, spatial learning and memory in light-dark cycle $12 / 12$ were significantly better than the other two groups, and spatial learning and memory are important indexes of cognitive function. This confirms again that CRDs can lead to cognitive decline. Third, compared with the other two groups BDNF in light-dark cycle $12 / 12$ is higher, and $A \beta$ in light-dark cycle $12 / 12$ lower. This suggested BDNF and $A \beta$ proteins were involved in the cognitive decline caused by CRDs [30].

There are many studies on the correlation between BDNF and $A \beta$ [8-13]. But in CRDs, there was few. It is need explored mechanisms of BDNF decreased and $A \beta$ increased in CRDs. It may be related to the following factors. First, alterations in the suprachiasmatic nucleus and melatonin secretion are the major factors linked with the cause of CRDs [31]. Adverse, CRDs cause alterations in the suprachiasmatic nucleus and melatonin secretion, and other hypothalamic hormone secretion abnormalities [31]. These hormones can affect brain cell function, including BDNF, amyloid precursor protein (APP) and metabolism of $A \beta$ precursor protein and key enzyme starch. This can lead to a decrease in BDNF and an increase in $A \beta$ [31]. Second, these hormones regulate a variety of body signs, such as blood sugar, lipids and blood pressure, these can indirectly lead to a decrease in BDNF and an increase in $A \beta[32$ 34]. Third, CRDs and emotional reactions such as depression and anxiety are mutually causal. Depression and anxiety are high risk factors for Alzheimer's disease, decrease in BDNF and an increase in $A \beta$ are characteristic changes of Alzheimer's disease [35].

There are some obvious limitations in this study. First, in this study, only the cognitive function and $A \beta$, BDNF level in hippocampus of mice with circadian rhythm disorder were detected. No other relevant indicators were detected however, this may explain part of the reason why CRDs lead to cognitive decline. Second, circadian rhythm disorder hormones secreted by hypothalamus in circadian rhythm disorder were not detected, but this had been confirmed by previous studies.

\section{Conclusion and Future directions}

Our study confirmed that changes in hippocampal function may be caused by light-dark cycles of different lengths in CRDs, BDNF decreased and increased $A \beta$ increased in hippocampal tissue. CRDs lead to cognitive function declines though BDNF decreased and increased $A \beta$ increased in hippocampal tissue. In the future, the molecular mechanism of cognitive dysfunction, BDNF decreased and increased $A \beta$ increased in hippocampal tissue caused by CRDs will be further studied. The elucidation of these mechanisms may provide new methods for clinical practice. it can reduce the incidence and prevalence of Alzheimer's disease and cognitive impairment caused by CRDs.

\section{Acknowledgement}

The authors thank the staff of the the State Key Laboratory of Management and Control for Complex Systems institute of Automation, Key Laboratory of Chronobiology of Health Ministry in Basic and Forensic School of Sichuan University, and the Department of Geriatrics of the third hospital of Mianyang, and all study participants (as well as their legal proxies) for their great contributions.

\section{Disclosure Statement}

The authors wish to extend their full confidence that there are no conflicts of interest in this research article and that only the fullest integrity was practiced in its composition.

\section{References}

1. Logan S, Owen D, Chen S, Chen WJ, Ungvari Z, et al. (2018) Simultaneous assessment of cognitive function, circadian rhythm, and spontaneous activity in aging mice. Gero Science 40(2): 123-137.

2. DA Weldemichael, GT Grossberg (2010) Circadian Rhythm Disturbances in Patients with Alzheimer's Disease: A Review. Int J Alzheimers Dis 2010: 716453.

3. Jan Homolak, Monika Mudrovčić, Barbara Vukić, Karlo Toljan (2018) Circadian Rhythm and Alzheimer's Disease. Med Sci (Basel) 6(3): 52.

4. Abigail J Hiller, Makoto Ishii (2018) Disorders of Body Weight, Sleep and Circadian Rhythm as Manifestations of Hypothalamic Dysfunction in Alzheimer's Disease. Front Cell Neurosci 12: 471.

5. Yiwei Cao, Rui-Hong Wang (2017) Associations among Metabolism, Circadian Rhythm and Age-Associated Diseases. Aging Dis 8(3): 314333.

6. Melissa Wilking, Mary Ndiaye, Hasan Mukhtar, Nihal Ahmad (2013) Circadian Rhythm Connections to Oxidative Stress: Implications for Human Health. Antioxid Redox Signal 19(2): 192-208.

7. Dariush FARHUD, Zahra ARYAN (2018) Circadian Rhythm, Lifestyle and Health: A Narrative Review. Iran J Public Health 47(8): 1068-1076.

8. Antonella Caccamo, Monica A Maldonado, Alex F Bokov, Smita Majumder, Salvatore Oddo (2010) CBP gene transfer increases BDNF levels and ameliorates learning and memory deficits in a mouse model of Alzheimer's disease. Proc Natl Acad Sci U S A 107(52): 22687-22692.

9. Maria Laura Giuffrida, Agata Copani, Enrico Rizzarelli (2018) A promising connection etween BDNF and Alzheimer's disease. Aging (Albany NY) 10(8): 1791-1792.

10. Zhi-Hao Wang, Jie Xiang, Xia Liu, Shan Ping Yu, Fredric P Manfredsson, et al. (2019) Deficiency in BDNF/TrkB Neurotrophic Activity Stimulates $\delta$-Secretase by Upregulating C/EBP $\beta$ in Alzheimer's Disease. Cell Rep 28(3): 655-669.

11. Huaiqing Luo, Yang Xiang, Xiangping Qu, Huijun Liu, Chi Liu, et al. (2019) Apelin-13 Suppresses Neuroinflammation Against Cognitive Deficit in a Streptozotocin-Induced Rat Model of Alzheimer's Disease Through Activation of BDNF-TrkB Signaling Pathway. Front Pharmacol 10: 395.

12. Jung Hoon Koo, In Su Kwon, Eun Bum Kang, Chang Kuk Lee, Nam Hee Lee, et al. (2013) Neuroprotective effects of treadmill exercise on BDNF and PI3-K/Akt signaling pathway in the cortex of transgenic mice model of Alzheimer's disease. J Exerc Nutrition Biochem 17(4): 151-160.

13. Henrique Cerqueira Guimarães, Paulo Caramelli, Patricia Paes Araujo Fialho, Elisa de Paula França, Marcelo Pelizzaro Dias Afonso, et al. (2013) Serum levels of soluble TNF- $\alpha$ receptors but not BDNF are associated with apathy symptoms in mild Alzheimer's disease and amnestic mild cognitive impairment. Dement Neuropsychol 7(3): 298-303. 
14. Rohe M, Synowitz M, Glass R, Paul SM, Nykjaer A, et al. (2009) Brainderived neurotrophic factor reduces amyloidogenic processing through control of SORLA gene expression. J Neurosci 29(49): 15472-15478.

15. Michael Rohe, Daniela Hartl, Anja Nawarecki Fjorback, Joachim Klose, Thomas E Willnow (2013) SORLA-Mediated Trafficking of TrkB Enhances the Response of Neurons to BDNF. PLoS One 8(8): e72164.

16. Magdalena Miranda, Juan Facundo Morici, María Belén Zanoni, Pedro Bekinschtein (2019) Brain-Derived Neurotrophic Factor: A Key Molecule for Memory in the Healthy and the Pathological Brain. Front Cell Neurosci 13: 363.

17. Shiyong Peng, Diego J Garzon, Monica Marchese, William Klein, Stephen D Ginsberg, et al. (2009) Decreased Brain-Derived Neurotrophic Factor Depends on Amyloid Aggregation State in Transgenic Mouse Models of Alzheimer's Disease. J Neurosci 29(29): 9321-9329.

18. Alexander Hawlitschka, Andreas Were (2018) Experimental Intrastriatal Applications of Botulinum Neurotoxin-A: A Review. Int J Mol Sci 19(5): 1392.

19. Kitiyanant N, Kitiyanant Y, Svendsen CN, Thangnipon W (2012) BDNF, IGF-1- and GDNF-secreting human neural progenitor cells rescue amyloid $\beta$-induced toxicity in cultured rat septal neurons. Neurochem Res 37: 143-152.

20. Moira S Lewitt, Gary W Boyd (2019) The Role of Insulin-Like Growth Factors and Insulin-Like Growth Factor-Binding Proteins in the Nervous System. Biochem Insights 12: 1178626419842176.

21. K Schindowski, K Belarbi, L Buée (2008) Neurotrophic in Alzheimer's disease: role of axonal transport. Genes Brain Behav 7(1): 43-56.

22. Liqi Tong, Robert Balazs, Rungtip Soiampornkul, Wipawan Thangnipon, Carl W Cotman (2008) Interleukin-1ßimpairs brain derived neurotrophic factor-induced signal transduction. Neurobiol Aging 29(9): 1380-1393.

23. Girardin Jean Louis, Ferdinand Zizi, Douglas R Lazzaro, Arthur H Wolintz (2008) Circadian rhythm dysfunction in glaucoma: A hypothesis. Circadian Rhythms 6: 1.

24. Joseph Nunez (2008) Morris Water Maze Experiment. J Vis Exp (19): 897.

25. Ahsan Habib, Darrell Sawmiller, Song Li, Yang Xiang, David Rongo, et al. (2017) LISPRO mitigates $\beta$-amyloid and associated pathologies in Alzheimer's mice. Cell Death Dis 8(6): e2880.
26. Mirian David Marques (2013) Biological rhythms and vector insects. Mem Inst Oswaldo Cruz 108(Suppl 1): 59-62.

27. Maria Angeles Bonmati-Carrion, Raquel Arguelles-Prieto, Maria Jose Martinez-Madrid, Russel Reiter, Ruediger Hardeland, et al. (2014) Protecting the Melatonin Rhythm through Circadian Healthy Light Exposure. Int J Mol Sci 15(12): 23448-23500.

28. Punyakishore Maibam, Ganesh M. Nawkar, Joung Hun Park, Vaidurya Pratap Sahi, Sang Yeol Lee, et al. (2013) The Influence of Light Quality, Circadian Rhythm, and Photoperiod on the CBF-Mediated Freezing Tolerance. Int J Mol Sci 14(6): 11527-11543.

29. Agnieszka Kiełbowicz-Matuk, Pascal Rey, Tadeusz Rorat (2014) Interplay between circadian rhythm, time of the day and osmotic stress constraints in the regulation of the expression of a Solanum Double B-box gene. Ann Bot 113(5): 831-842.

30. Nicola Solari, Balázs Hangya (2018) Cholinergic modulation of spatial learning, memory and navigation. Eur J Neurosci 48(5): 2199-2230.

31. Pamela Newland, Angela Starkweather, Matthew Sorenson (2016) Central fatigue in multiple sclerosis: a review of the literature. J Spinal Cord Med 39(4): 386-399.

32. A B Davies, B A Gould, P M Cashman, E B Raftery (1984) Circadian rhythm of blood pressure in patients dependent on ventricular demand pacemakers. Br Heart J 52(1): 93-98.

33. C Maggioni, G Cornélissen, K Otsuka, F Halberg, D Consonni, U Nicolini (2005) Circadian rhythm of maternal blood pressure and fetal growth. Biomed Pharmacother 59(Suppl 1): S86-S91.

34. Wen Su, Zhenheng Guo, David C Randall, Lisa Cassis, David R Brown, et al. (2008) Hypertension and disrupted blood pressure circadian rhythm in Type 2 diabetic db/db mice. Am J Physiol Heart Circ Physiol 295(4): H1634-H1641.

35. Randy F Crossland, Alfred Balasa, Rajesh Ramakrishnan, Sangeetha K Mahadevan, Marta L Fiorotto, Ignatia B Van den Veyver (2018) Chronic Maternal Low-Protein Diet in Mice Affects Anxiety, Night-Time Energy Expenditure and Sleep Patterns, but Not Circadian Rhythm in Male Offspring. PLoS One 13(7): e0201079. 